\title{
Sacroiliac Joint Syndrome
}

To the Editor:

I enjoyed reading the April 2001 issue of Pain Physician. I have a few comments.

The Sacroiliac Joint Syndrome (SIJS) article by Slipman et al (Pain Physician 2001; 4:97-100) was informative and interesting. However, I still wonder if we do have a "gold standard” with intraarticular injection. The volume of local typically used makes the block non-specific to joint, ligament or perhaps even nerve branches. Confirming intraarticular placement is frequently questionable. The closest it comes to this "gold standard" is after facets and discs have been excluded. After Caragee's debatable findings of false positive discs with iliac crest pain generator could there be false positive discs for SIJ generator or visa versa? A "gold standard" is a test that to me has a very high true positive rate and very low false negative rate. At this point it doesn't appear that there is significant confidence in intraarticular SIJ inj.

The article on the cervical selective nerve root blocks (SNRB) in whiplash by Slipman et al (Pain Physician 2001; 4:97-100) was interesting. It must be read with caution. In the wrong hands this article is going to be concluded to read "there is no point performing SNRB/TF ESI on whiplash patients. In reality, the conclusions is that "in the absence of MRI findings of disc pathology or objective signs of radiculopathy, SNRB/TF ESI is ineffective for whiplash patient. It is still a highly effective treatment for patients with true radiculopathy and in patients with disc herniations."
Joseph F. Jasper, MD

Medical Director

Advanced Pain Medicine Physicians

1628 South Mildred Street, Suite \#105

Tacoma, WA 98465-1613

In Response:

Dr. Jasper raises numerous issues about two articles published from the Penn Spine Center, which are best addressed in a sequential manner. Due to space limitations I will respond to most but not each of the non-sequiturs.

It is reasonable to state the diagnostic sacroiliac joint block currently is the "gold standard" diagnostic tool from which a diagnosis of sacroiliac joint syndrome can be made. Such a notion is supported by conclusions formulated after examination of two independent issues. First, there is neither another diagnostic tool including history, examination, imaging nor intraoperative findings that provides better information to make this diagnosis. Second, therapeutic outcomes of a specific intervention for this disorder can be determined as they relate to a single or double diagnostic block response. Therefore, even in the event that this diagnostic study is not perfect, rational treatment decisions can be made and prognoses offered. Such analyses has led to the widespread acceptance that diagnostic sacroiliac joint block is the gold standard test. Obviously this analysis does not imply that it is an ideal test or that a better diagnostic tool is not desired. A good analogy would be the state of care provided to patients on the basis of myelogra- 
phy before the advent of CT scan or MRI. Rational decisions were made incorporating an understanding of the limitations of that diagnostic tool. By the way, despite what the term "gold standard" means to Dr. Jasper, for the rest of us it denotes the diagnostic test against which all other diagnostic tests/interventions are judged.

While I appreciate Dr. Jaspers difficulty confirming intrarticular placement, and I am assuming he refers to local anesthetic flow rather than needle tip location, this ought not lead to the erroneous conclusion that all spine interventionalists are confronted with the same problem. During my nearly 10 year experience teaching extremely bright and gifted interventional physiatric fellows this has been a common problem for each of them during the first 3 to 9 months of training. Upon learning the variety of approaches for SIJ injection this issue of "am I in?” becomes a non-issue.

Dr. Jasper queries whether there are patients with intradiscal pain who may have reported false positive SIJ block responses. It is unclear why this question is raised, since it was answered quite clearly in our article. The answer is yes! Another reading of the paragraph concerning our diagnostic and therapeutic algorithm addresses this concern head on. In fact, our algorithm is derived to specifically deal with that potential problem.

Dr. Jasper suggests that our article on whiplash induced cervical radicular pain could be misinterpreted by an unwary or unsophisticated individual to erroneously conclude that Selective Nerve Root Block is ineffective for this particular disorder. His concern stems from his belief that it is an effective treatment for patients with "true radiculopathy and disc herniations". We never made a final judgment that this procedure is ineffective for whiplash induced cervical radicular pain. Rather we reported our preliminary results and emphasized the need for further study. Such a conclusion was made because of our small sample size and the absence of a control population. However any reasonable physician should surmise that a procedure with less than $20 \%$ good and excellent results does not seem to be a useful intervention in the patient population we analyzed. I understand the frustration experienced when scientific inquiry does not provide factual support for treatments we would like to offer patients despite our firm, myth based, convictions. Yet, it is our ability to garner and assimilate this information in a manner that alters our evaluative and treatment processes that distinguishes physicians from gurus. We made no commentary concerning this procedure for patients with symptoms of a herniated nucleus pulposis. Although, we believe such injection is appropriate for this disorder no study has proven this assumption.

Curtis W. Slipman, MD

Director, Penn Spine Center

Chief, Division of Musculoskeletal Rehabilitation

Associate Professor of Rehabilitation Medicine

Associate Professor of Orthopedic Surgery 